\title{
Hydraulic characteristics of internal protective coatings used for rehabilitation of pipelines of water supply and sanitation systems
}

\author{
Vladimir Orlov ${ }^{1 *}$ \\ ${ }^{1}$ Moscow State University of Civil Engineering, 26, Yaroslavskoe Shosse, Moscow, 129337 \\ Russia
}

\begin{abstract}
The purpose of this article is to provide a comparative analysis of hydraulic characteristics of some modern organic coatings used for trenchless rehabilitation of engineering pipelines. Scotchkote 169HB pipeline inner protective coating manufactured by « $3 \mathrm{M} »$ production company (USA) and Subcote FLP coating by «Group POLYPLASTIC» Ltd (RF) were the objects of this investigation. The article presents general characteristics of alternative protective coatings. A methodical approach to determination of the protective coating hydraulic characteristics is described by the tests at a special patented small-sized bench through estimation of the coatings' hydrophoby with the terms of hydraulic characteristics. Based on the obtained results the conclusions are made concerning the feasibility of application of organic coatings compared to non-organic ones (for example, cement-sand coatings). This will enable power savings due to a lower hydraulic resistance during the water transportation. Based on the experimental studies in static and dynamic conditions on a small-sized testing bench it was proved, that the Subcote FLP protective coating has hydrophilic properties. There are shown results of an automated processing of experimental data and comparison of the values of the coefficients of hydraulic friction, resistivity and roughness, which have been obtained at the large-sized and small-sized benches, as well as at a roughness indicator. Provision has been made of a possible and reasonable use of small-sized benches instead of large-sized ones for determination of hydrophoby and hydraulic parameters, which will considerably facilitate the traditional method of comprehensive estimation of the hydraulic performance of various types of protective coatings.
\end{abstract}

\section{Introduction}

By the XXI century, the RF cities have faced the poor state of underground pipeline systems of water supply and sanitation, where more than $60 \%$ of the pipelines need an urgent repair and modernization with a wide use of trenchless technologies [1]. The trenchless rehabilitation of the pipeline transportation is subject to different technologies, which include pulling of round and end-section deformed polymer pipes [2], use of

\footnotetext{
*Corresponding author: orlov950@yandex.ru
} 
polymeric sleeves and application of inner spiral wrapping of infinite polymeric ribbons at the inner surface of pipelines [3, 4]. Protective coatings, which are applied to the inner surface of the pipelines by the centrifugal spraying, are one of the perspective methods of the trenchless pipeline rehabilitation. These coatings include non-organic materials on the basis of construction cement-sand mortars, which have been practiced in the pipeline rehabilitation for nearly 100 years, as well as new types of coatings, which are classified as organic ones [5]. Organic coatings are much more advantageous than non-organic ones due to some higher strength characteristics and lower hydraulic resistances, which increase the power saving effect during the water flowing $[6,7]$.

Protective coatings, which are used in the trenchless rehabilitation works, shall provide solution of the resource saving problems in the flow transportation pipelines both of natural and sewage water, i.e. enable creation of the conditions of accident-free mode of operation of engineering networks through efficient and, to the extent possible, quick repair of all types of defects on pipelines.

The actual construction market proposes a wide range of the pipeline protective coatings. However, before a proposal is made of application of such or another material for rehabilitation of the pipelines, the proposed coating shall be tested to estimate its strength and hydraulic properties [8].

\section{Literature review}

The following information is given herein as a brief characteristic of the above-mentioned coatings. Over the last decade in European countries and the United States the internal protective coating Scotchkote 169HB $[9,10]$ is widely applied in water supply systems for pipeline rehabilitation. It has high strength characteristics, but the technical literature does not provide data on its hydraulic performance, or these data are contradictory [11]. The material of the coating is $100 \%$ insoluble aliphatic isocyanate polyurethane. The coating is applied to the inner surface of the pipelines by centrifugal spraying in the range of 100$1000 \mathrm{~mm}$ diameters. The coating thickness for a single application can be up to $5 \mathrm{~mm}$ (typically, a layer is $3 \mathrm{~mm}$ thick). Gelation time (at $25^{\circ} \mathrm{C}$ ) is 1 minute; the formation of a film takes 2 minutes and polymerization on the inner surface of the pipe - about 10 minutes.

To the next generation of protective coatings company «3M» should be attributed Scotchkote 2400 , which is a rapidly solidifying polyurea. The coating is applied in the process of centrifugal spraying. The coating composition is a two-component one, where the basic component is a white thixotropic liquid and the thixotropic black liquid is an activator. The maximum thickness of the coating when applied to a single layer is $2.75 \mathrm{~mm}$. When such a coating thickness is in the range of $100-1000 \mathrm{~mm}$ diameters, it is able to fill in the corrosion holes (fistulas) and crevices (cracks), annular gaps (for example, stretched joints, annular tears) and other similar defects up to $5 \mathrm{~mm}$ size. The internal surface roughness of the restored pipeline is reduced, which is reflected in the flow rate profiles [12]. The gelatinization time is 60 seconds, the formation of the film makes 3 minutes, and the polymerization time takes about 60 minutes.

A relatively new type of protective coatings is a material called Subcote FLP, developed by «Group POLYPLASTIC» Ltd $[13,14]$. It is a quickly solidifying polyurethane resin, which is sprayed on the inner surface of steel, iron, concrete and asbestos cement pipes with a nominal diameter from 100 to $1200 \mathrm{~mm}$. The composition of Subcote FLP coating is a two-component one: the basic component is a pale blue liquid; the activator is a brown liquid. The maximum thickness of the coating when applied to a single layer is $4 \mathrm{~mm}$. When the thickness of the protective coating is $3 \mathrm{~mm}$ it is able to block the through holes (fistula) with a diameter up to $8 \mathrm{~mm}$, as well as circumferential and longitudinal cracks. The 
gelatinization time is 32 seconds, formation of a stable film makes 6 minutes, and the polymerization time with the possibility of CCTV inspection is 60 minutes.

\section{Materials and methods}

Experimental study of the hydraulic properties of the materials of Scotchkote 169HB, Scotchkote 2400 and Subcote FLP coatings were made in the laboratory of the Department "Water supply and Sewerage" of NIU MGSU in the years 2016-2017. The aim of the protective coating investigation was to obtain the hydraulic characteristics of protective coatings, which may be used by a designer, in particular: the coefficient of equivalent roughness $\mathrm{k}$, of the hydraulic friction coefficient $\lambda$ and the ratio of the resistivity $A$. The hydraulic studies were made on two benches and were compared with results of experiments aimed at determining the roughness, got by a TR 1000 roughness indicator. The data, which have been got at the roughness indicator, were used in the automated software in the simulation series aimed at determining hydro-mechanical values of protective coatings.

To determine the hydraulic characteristics provision was made both of the traditional methods and the improved ones based on the use of a patented test equipment for investigation of the protective coating hydrophoby. For the general view of the experimental bench see Fig. 1, the diagram of the equipment for traditional hydraulic tests is given in Fig. 2.

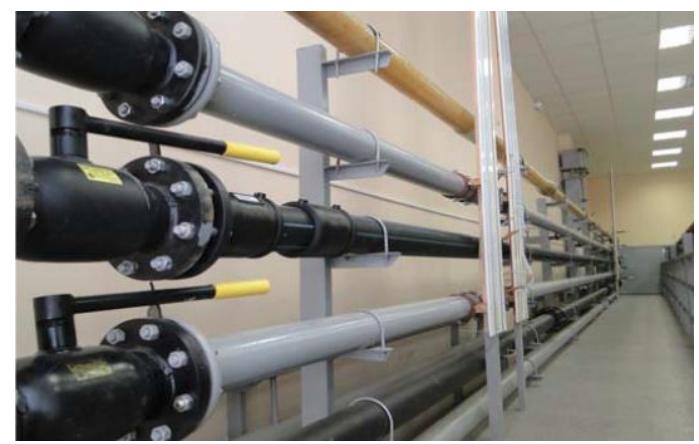

Fig. 1. General view of the hydraulic bench with pipes made of different materials

The experimental hydraulic test bench has the following main elements: a storage tank with a capacity of $4 \mathrm{~m}^{3}$; the trestle with $15 \mathrm{~m}$ long pipelines which have a protective coating and are rigidly fixed on it, a pumping unit on the base of a multistage pump Grundfos CRE 90$2-2$, with a capacity of $90 \mathrm{~m}^{3} / \mathrm{h}, 30 \mathrm{~m}$ pressure and a motor of $11 \mathrm{~kW}$ power and $360-$ 3,600 revolutions per minute; ultrasonic flow meter US-800. As an apparatus for measuring the pressure of water in the pipeline use has been made of the roughness indicators spaced at the distance of $10 \mathrm{~m}$ (the pipeline operation part) between them. The above described bench has been used for the hydraulic experiments with Scotchkote 169HB, Scotchkote 2400 coatings.

The experiments with Subcote FLP coating were carried out both at the aforementioned large-sized bench, and a special compact test stand for determination of the hydrophoby degree and the hydraulic performance indices [15]. The target of the parallel experiments was a comparison of the received hydraulic characteristics of the coating to examine possible refuse from large-sized installations and possible future works on compact benches, when it is necessary to carry out hydraulic experiments with new protective coatings. 


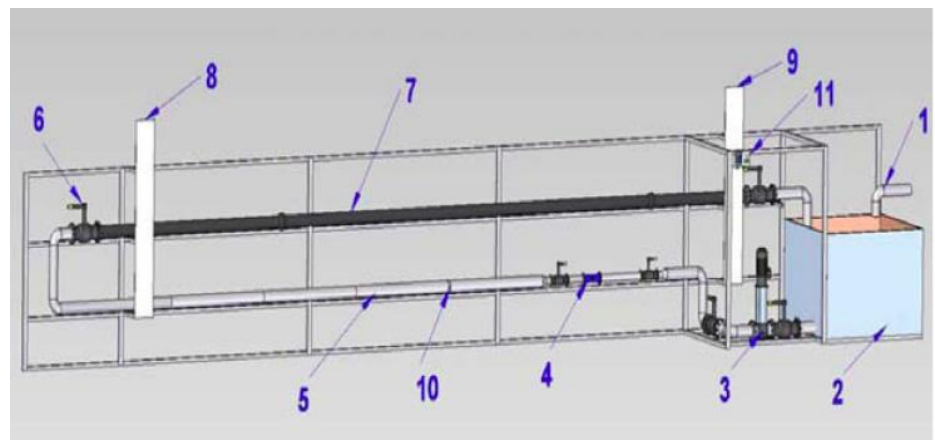

Fig. 2. General scheme of the equipment for hydraulic tests of sprayed protective coatings: 1 water supply; 2 - storage tank; 3 - pump; 4 - sensor of a flowmeter; 5 - feed pipe; 6 - valve; 7 pipeline coated with a protective coating; 8,9 - bench with roughness indicators; 10 - mounting frame for the pipeline fixation; 11 - ultrasonic flow meter

The proposed method of the study was to investigate aspects of the interaction of the liquid with the surface of the protective coating, namely:

-behavior of water drops (no more than $0.55 \mathrm{ml}$ ) in statics on the surface of the protective coating;

-behavior of the flowing stream in dynamics, if it flows by a sloped surface with the protective coating in the form of a chute (Fig. 3 and Fig. 4).

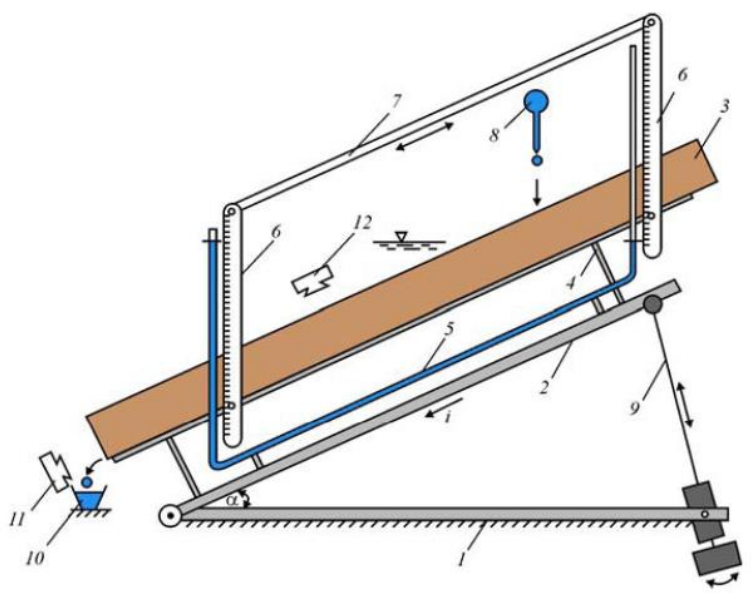

Fig. 3. Schematic drawing of the experimental equipment at the maximal slope of a $1 \mathrm{~m}$ long chute: 1 - support frame; 2- rack; 3 - an open chute (tray); 4 - posts; 5 - the system of communicating vessels; 6 -measuring rules; 7 - the control bar; 8 - pipette with water; 9 - rod mechanical Jack; 10 a collection of drops; 11, 12 -respectively, the camera of frontal and coaxial front shooting of the flow

The Fig. 5 shows a design in the form of an open chute made of polyvinyl chloride of $130 \mathrm{~mm}$ rigidly attached Subcote FLP coating with a thickness of $1.1 \mathrm{~mm}$. The drinking water was used as a liquid for formation of droplets and a mini-flow (or mini-strim) in the chute to simulate operation of diameter with a liquid transfer gravity pipeline.

As the instrumentation for photo and filming of the drop configuration and the liquid mini-flow use has been made of a digital SLR camera Sony 550, the lens is DT 1.8/50 SAM in complex with extension tubes Kenko Extension tube with continuous multi-frame and a digital video camera Sony model HDR-CX250E. 
The methods of static studies of the protective coating Subcote FLP on a small-sized bench were aimed at determining the degree of wettability of the protective coating, namely, the contact angle $\alpha$ of the spreaded drop and the mini-flow. This indicator is a criterion for a classification of the protective coating to the category of hydrophobic $(\alpha>$ $\left.90^{\circ}\right)$ or hydrophilic $\left(\alpha<90^{\circ}\right)$ one [16].

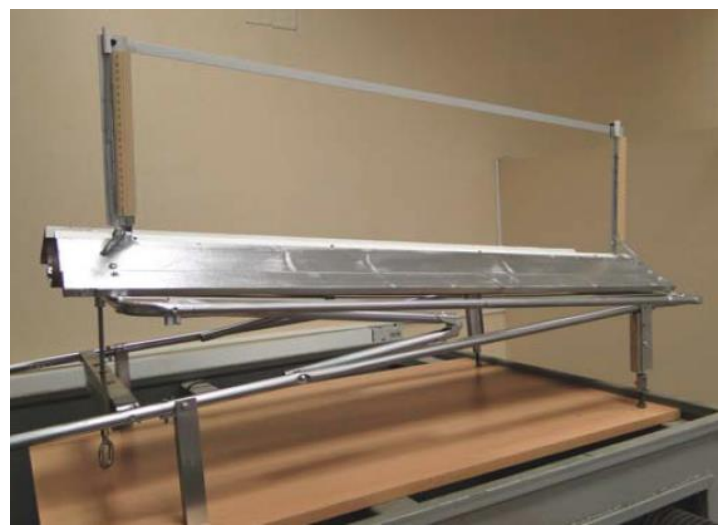

Fig. 4. Experimental equipment for the study of water hydrophoby in nature

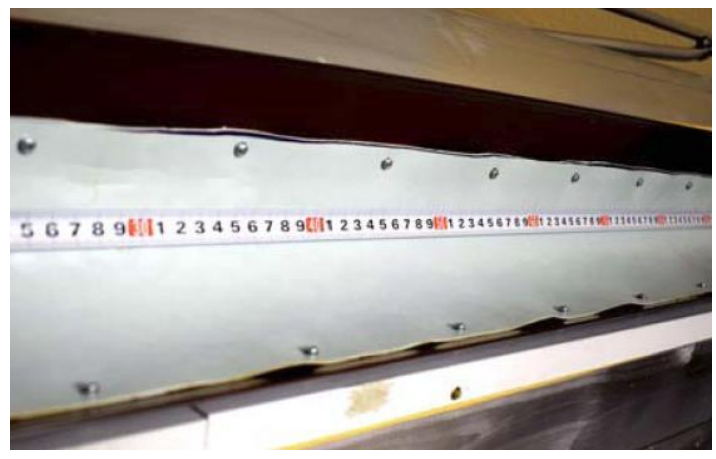

Fig. 5. Fragment of the experimental equipment with an open chute, and the applied coating Subcote FLP

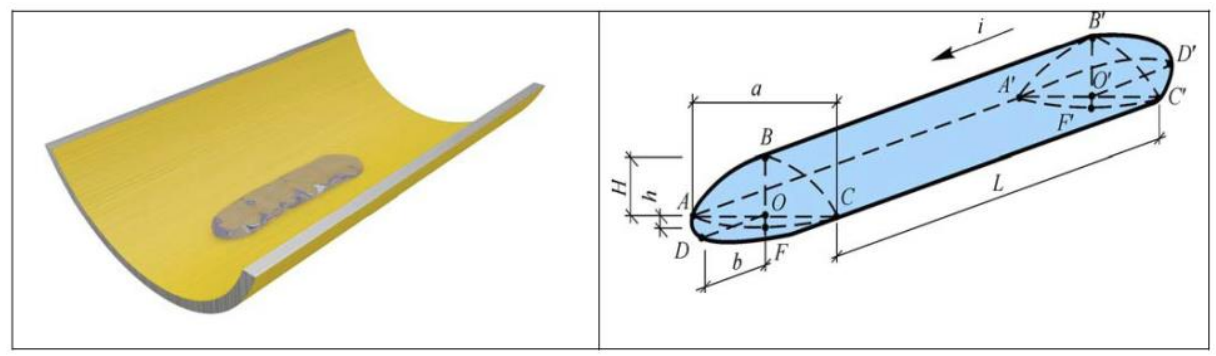

Fig. 6. Axonometric diagram of the mini-flow generated due to the flow of the critical volume of the liquid along a sloped chute with the protective coating Subcote FLP in the form of a tray of $1 \mathrm{~m}$ length: a, L ,respectively, - average values of the width and length of the compact part of mini-flow; $\mathrm{H}, \mathrm{h}$, respectively, the heights of the upper and lower lenses; $\mathrm{b}$ - height of the half of the ball segment of the mini-flow head part; $\mathrm{AFC}, \mathrm{AA}{ }^{\prime} \mathrm{C}^{\prime} \mathrm{C}$ respectively- the wetted perimeter and the surface of the compact part of mini-flow; $\mathrm{i}$ - slope of the chute. 
In turn, the technique of dynamic research was to study the formation and spontaneous movement of the liquid mini-flow in the chute due to its appropriate slope and volume of the liquid critical mass. General view of the mini-flow at a slope I of the working surface is shown in Fig. 6. The head and the tail part of the mini-flow are presented in the form of a three-dimensional structure (half of a ball segment), respectively $A B C D A$ and $A^{\prime} B^{\prime} C^{\prime} D^{\prime} A$ '. Their projections on the vertical plane can be represented in the form of a lens (the circumference) by analogy with front projection of the drops.

Determination of the geometric dimensions of the mini-flow was carried out by the front and coaxial shooting, a mathematical description of the mini-flow element shape: the heights of the top lens $\mathrm{H}$ and the bottom lens $\mathrm{h}$, the equation of the top of the lens and a tangent thereto at the point of intersection with the horizontal plane, the boundary angles $\alpha$, the areas of the projections of the lenses to the vertical plane (for example, to the top of the lens $S v=2 a H / 3$ ) and other parameters, allowing to estimate the degree of hydrophoby of the material and if possible to identify its relationship with geometric figures of the mini-flow.

\section{Results}

Mathematical processing of results of tests of the structure «steel pipe + protective coating Subcote FLP» on a long bench with an inner diameter of $0.094 \mathrm{~m}$ and a layer thickness of $2.5 \mathrm{~mm}$ showed that the average value of the hydraulic friction coefficient is $\lambda \mathrm{FLP}=0.01772$, the structure «steel pipe + protective coating Scotchkote $169 \mathrm{HB}$ » with an inner diameter of $0.0952 \mathrm{~m}$ and thickness $2.4 \mathrm{~mm}-\lambda 169 \mathrm{HB}=0.01925$ and the structure «steel pipe + protective coating Scotchkote 2400» with an inner diameter of $0.0952 \mathrm{~m}$ and thickness $2.4 \mathrm{~mm}-\lambda 12400=0.01959$. Fig. 7 shows a photograph of drops on the surface of the protective coating Subcote FLP under static conditions.

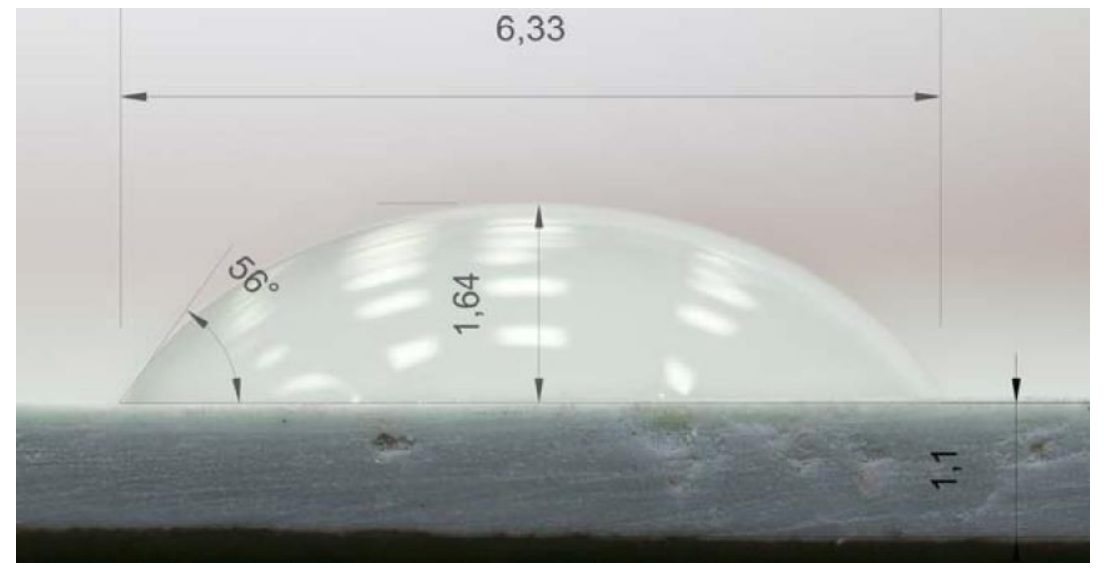

Fig. 7. Photograph of a drop on the horizontal surface of the protective material Subcote FLP with the edge angle $56^{\circ}$

The geometric dimensions of the diameter size of the spreading of the drop on the surface $(6.63 \mathrm{~mm})$ and the maximum height of the drop $(1.64 \mathrm{~mm})$ were determined from the photographs of frontal projection of drops on the protective coating. The results of the experiments the contact angle was $56^{\circ}(<90$ degrees), indicating the hydrophilic nature of the protective coating Subcote FLP.

The fragments of the frontal and coaxial filming of the mini-flow in a chute with Subcote FLP protective coating under dynamic conditions are presented in Fig. 8. 

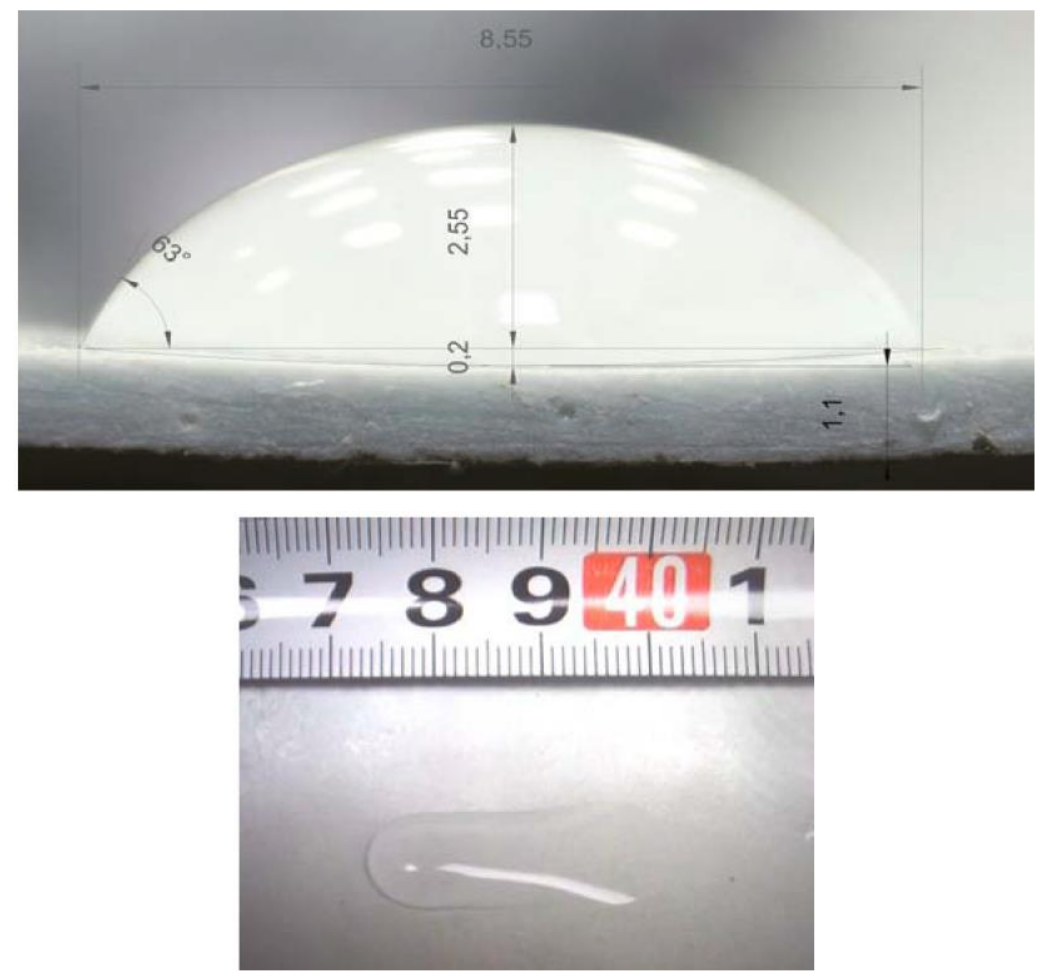

Fig. 8. Selective fragments of frontal (top) and coaxial (bottom) photos of the flow

According to the results of the performed experiments on the TR 100 roughness indicator, the following data were obtained on the average roughness of the protective coatings:

-for Scotchkote 169HB: $k_{169}=3.50 \mathrm{mkm}=0.0385 \mathrm{~mm}$

-for Scotchkote 2400: $k_{2400}=m k m$ of $45.57=0.04557 \mathrm{~mm}$

-for Subcote FLP: $k_{\mathrm{FLP}}=36.80 \mathrm{mkm}=0.0368 \mathrm{~mm}$

\section{Discussion}

\subsection{Interpretation of results of hydraulic experiments on a large-size bench}

To determine the values of the $A$ specific resistances obtained on the hydraulic bench the data were processed using a computer program which supports the hydraulic study of pressure pipelines. The program allows the calculation of the hydraulic parameters and performance of hydro-mechanical modeling of pipelines with the change of their diameters $\mathrm{d}$ in obtaining the dependence $A=f(d)$. According to the results of the computer processing of the experiments' results on the hydraulic stand the mathematical dependence of $A=f(d)$ type has been got, as well as the values of specific resistances $A$ for a wide range of diameters of the pipelines with applied protective coatings (in the range of $0.1-1.2 \mathrm{~m}$ ). The results of the processing are presented in the Table 1 . It also includes values of the specific resistance of the pipes coated with cement-sand coating. 
Table 1. Calculated values of specific resistance of pipelines of various diameters $\mathrm{c}$ applied protective coatings

\begin{tabular}{|c|c|c|c|c|c|}
\hline \multirow{4}{*}{ No } & \multirow{4}{*}{$\begin{array}{c}\text { Inner } \\
\text { diameter of } \\
\text { the pipe } d, m\end{array}$} & Subcote FLP & $\begin{array}{c}\text { Scotchkote } \\
169 \mathrm{HB}\end{array}$ & $\begin{array}{c}\text { Scotchkote } \\
2400\end{array}$ & \multirow{3}{*}{$\begin{array}{c}\text { Cement-sand } \\
\text { coating }\end{array}$} \\
\hline & & $\mathrm{A}_{\mathrm{FLP}}=0.0008 \mathrm{~d}^{-}$ & $\begin{array}{c}\mathrm{A}_{169}=0.0008 \mathrm{~d}^{-} \\
5.19\end{array}$ & $\mathrm{~A}_{2400}=0.00095 \mathrm{~d}^{-}$ & \\
\hline & & $\begin{array}{c}\text { K FLP }=0.0368 \\
\text { mm }\end{array}$ & $\begin{array}{c}\text { k } 169=0.0385 \\
\text { mm }\end{array}$ & $\begin{array}{c}\text { k } 2400=0.04557 \\
\text { mm }\end{array}$ & \\
\hline & & \multicolumn{4}{|c|}{ Specific resistance values $A, \mathrm{~s}^{6} / \mathrm{m}^{2}$} \\
\hline 1 & 0.1 & 123.336036 & 123.905330 & 147.137579 & 322.91 \\
\hline 2 & 0.15 & 15.049724 & 15.106935 & 17.939485 & 33.49 \\
\hline 3 & 0.2 & 3.383346 & 3.394254 & 4.030676 & 6.048 \\
\hline 4 & 0.25 & 1.063108 & 1.066059 & 1.265945 & 1.841 \\
\hline 5 & 0.3 & 0.412843 & 0.413838 & 0.491433 & 0.7374 \\
\hline 6 & 0.35 & 0.185552 & 0.185942 & 0.220806 & 0.3192 \\
\hline 7 & 0.4 & 0.092812 & 0.092982 & 0.110416 & 0.1657 \\
\hline 8 & 0.45 & 0.050376 & 0.050456 & 0.059917 & 0.8533 \\
\hline 9 & 0.5 & 0.029163 & 0.029204 & 0.034679 & 0.04939 \\
\hline 10 & 0.6 & 0.011325 & 0.011337 & 0.013462 & 0.01951 \\
\hline 11 & 0.7 & 0.005090 & 0.005094 & 0.006049 & 0.009424 \\
\hline 12 & 0.8 & 0.002546 & 0.002547 & 0.003025 & 0.004784 \\
\hline 13 & 0.9 & 0.001382 & 0.001382 & 0.001641 & 0.002596 \\
\hline 14 & 1.0 & 0.000800 & 0.000800 & 0.000950 & 0.001503 \\
\hline 15 & 1.2 & 0.000311 & 0.000311 & 0.000369 & 0.0005833 \\
\hline
\end{tabular}

As follows from the Table 1 the resistivity values for three types of the coatings under investigation are approximately equal, whereas for cement-sand coating the resistivity is much higher (in the range of $2.2-1.55$ times). The got data may attest to the fact that for the resource and power efficiency during the water transportation by pressure pipelines it is more advantageous to apply organic coatings as they have a lower hydraulic resistance. Similar conclusions can be also made according to the values of the coefficients of the equivalent roughness $k$, where the Subcote FLP coating prevails.

The practical significance of the mathematical dependences $A=f(d)$, which have been got during the experiments, consists in their application in the hydraulic calculations during designing of the pressure pipeline systems. In particular, to determine the $H_{d}$ pressure loss in the pressure pipes of a $l_{d}$ given length coated on their inner surface by Subcote FLP, if the pipe flow pass $q$, the calculation may be made according to the formula (1):

$$
H_{d}=A l_{d} q 2
$$

where $H_{d}$ is the pressure loss, $m$ of water column; $A$ - coefficient of resistivity, $s^{2} / m^{6}$; $l_{d}$ - the length of the pipeline part, $m ; q$ - flow rate, $m^{3} / s$.

Using the got empirical dependence $A=f(d)$, the formula (1) takes the form (2):

$$
H_{d}=0.0008 d^{-5.188} l_{d} q^{2}[\mathrm{~m}]
$$

where $d$ is the inner diameter of the pipeline part, $\mathrm{m}$.

For example, when the inner diameter of the pipeline is $0.3768 \mathrm{~m}$ (including the thickness of the coating layer), the length makes $100 \mathrm{~m}$ and the flow rate is $0.3 \mathrm{~m}^{3} / \mathrm{s}$, the head losses $H_{d}$ will be:

$$
H_{d}=0.0008 d^{-5.188} l_{d} q^{2}=0.0008 \cdot 0.3768^{-5.188} \cdot 100 \cdot(0.3)^{2}=1,14 \mathrm{~m}
$$




\subsection{Interpretation of the results of experiments on small-sized bench for the study of hydrophoby}

The main conclusion according to the results of the static studies (see Fig. 7) is that the studied surface of the protective coating can be classified as hydrophilic one. Analysis of the photo in Fig. 8 shows that the dynamics show a similar trend, confirming the hydrophilic nature of the protective coating Subcote FLP (the contact angle $\alpha$ is $63^{\circ}$ ). Assessment of the degree of hydrophobicity in the dynamics was carried out by determining the relative coefficient of hydrophobicity $\mathrm{K}_{\mathrm{o}}(3)$ :

$$
K_{o}=S_{v} /(S \cdot i)
$$

where $S_{v}$ is the area of the projection of the upper lens ( $A B C O A$ in Fig. 6$), \mathrm{mm}^{2} ; \mathrm{S}-$ area of the wetting surface of the chute by the compact part of L mini-flow by the width of the arc (the wetted perimeter) $A F C, \mathrm{~mm}^{2} ; i$ is the slope of the chute.

Using the geometrical dimensions and the mini-flow average rates, which have been got during the experiments, whole range of necessary hydraulic parameters of the investigated protective coatings has been determined followed by statement of the roughness coefficient of the appropriate protective coating «n». The algorithm for the hydraulic parameter analysis consists in determination of: the coefficient of hydrophoby $K_{o}$, the mini-flow filling $(h / d)$ and the wetted perimeter (the length of the $\operatorname{arc} A F C$ ); the hydraulic radius $R$ in terms of filling and using the wetted perimeter and their comparison with the subsequent adjustment of the formula corresponding to the design filling; the mini-flow rate $V$, as the quotient of division of the path $U$ which has been passed by the mini-flow head for the time $t$ recorded by a stopwatch; the coefficient Chezie $\mathrm{C}\left(C=V /(R i)^{0,5}\right)$, which depends on the hydraulic radius $R$, the rate $V$ and the slope $i$; the coefficient of relative roughness «n» according to Manning $C=\frac{1}{n} \cdot R^{1 / 6}$

The processing of the experimental data on the study of the hydrophoby / hydrophily of the Subcote FLP coating has been made using a patented automated program «Hydrophoby» [17].

When performing the automated calculation 12 experimental and reference data were taken into account. It is conducive to a complex calculation of parameters of hydrophoby and determination of the hydraulic parameters with the subsequent determination of the coefficient of roughness «n» and the hydraulic friction coefficient $\lambda$. Below is the printout of the results of the automated calculation program «Hydrophoby» for Subcote FLP coating.

1. The total volume of the mini-flow $W_{l}, \mathrm{ml}: W_{l}=0,0568 N+0,4943=2.1983$

2. The mini-stream total volume $W, \mathrm{~mm}^{3}: W=10^{3} W_{l}=2198.3$

3. Top lens area $S_{v}, \mathrm{~mm}^{2}: S_{v}=2 a H / 3=14.535$

4. Bottom lens area $S_{n}, \mathrm{~mm}^{2}: S_{n}=2 a h / 3=1.14$

5. Small chord length $\mathrm{m}, \mathrm{mm}: m=\left[(a / 2)^{2}+h^{2}\right]^{1 / 2}=4.2797$

6. Wetted perimeter p, mm: $p=2 m+(2 m-a) / 3=8.5625$

7. Total area of the top and bottom lenses $S_{s}, \mathrm{~mm}^{2}: S_{s}=S_{n}+S_{v}=15.675$

8. Theoretical volume of the compact part of the mini-stream $W_{t}, \mathrm{~mm}^{3}: W_{t}=S_{s} L=423.225$

9. The wetted surface of the compact part of the mini-stream $S, \mathrm{~mm}^{2}: S=p L=231.1867$

10 . Relative index of hydrophobicity $K_{o}: K_{o}=S_{v} /(S i)=0.4191$

11. Edge angle (angle of wetting) $\alpha$, deg.: $\alpha=50.05$

12. The estimated volume of the mini-stream $W_{r}, \mathrm{~mm}^{3}: W_{r}=W-(1 / 12)\left[H_{g}\left(H_{g}{ }^{2}+0.75 a^{2}\right)+\right.$ $\left.H_{k}\left(H_{k}^{2}+0.75 a^{2}\right)\right]=423.225$

13. Estimated length of the mini-stream compact part $L_{r}, \mathrm{~mm}: L_{r}=W_{r} / S_{s}=136.4464$

14. The approximation between the projected length $L_{r}$ and the average length $L$ of the mini-stream $P_{L}, \%$ : 


$$
P_{L}=\left|100-L \cdot 100 / L_{r}\right|=8.212
$$

15. Tray filling $(h / d):(h / d)=h / d=0.0015385$

16. Hydraulic radius $R_{l}$ (through filling), $\mathrm{mm}: R_{l}=62.2(\mathrm{~h} / \mathrm{d})^{0,8833}=0.20377$

17. Hydraulic radius $R_{2}$ (through the wetted perimeter and live section), mm: $R_{2}=S_{n} / p=$ 0.13314

18. The absolute approximation value $P_{R}$ between $R_{I}$ and $R_{2}, \%: P_{R}=\left|100-R_{I} \cdot 100 / R_{2}\right|=$ 53.0517

19. The adjusted exponent « $x$ » in the formula to determine the hydraulic radius $R_{2}=$ $62.2(h / d)^{x}: \quad x=0.94901$

20. The general corrected formula for determining the hydraulic radius $R$ and its value, $\mathrm{mm}$ : $R=62.2(h / d)^{X}=62.2(h / d)^{0.94901}=0.13314$

21. Average mini-stream speed V, mm / s: $V=U / t=9.3069$

22. The Chezie's Coefficient $C: C=V /(R i)^{1 / 2}=65.85791$

23. Manning's relative roughness coefficient $n: n=(1 / C) R^{1 / 6}=0.0109$

24. Hydraulic friction coefficient $\lambda_{F L P}{ }^{*}:=8 g / C^{2}=89.81 / C^{2}=0.0181$

According to the results of the experiments which have been performed on the pipe study hydraulic bench and the plant for study of the hydrophoby degree of materials it shall be noted, that the convergence of the results is very high as for definition of the average value of the hydraulic friction coefficient $\lambda$.

The error in determining values for $\lambda$ was:

$$
\left|\left(\lambda_{F L P}-\lambda_{F L P}{ }^{*}\right) / \lambda_{F L P}{ }^{*}\right|=|(0,01772-0,0181) / 0,01772|=0,0214(2,14 \%)
$$

Thus, the results obtained by high convergence of $\lambda$ values confirmed possible refuse from a large-sized equipment and transition to small-sized benches for performance of the hydraulic experiments with protective coatings.

\section{Conclusions}

1. Hydraulic parameters of alternative organic protective coatings which can be used during trenchless rehabilitation of pressure pipelines to achieve the effect of energy saving have been determined and analyzed on special benches.

2. The methodology of field experiments to determine the degree of hydrophoby and hydraulic parameters has been developed for a small-sized testing bench under dynamic conditions by studying the behavior of a liquid mini-flow at a wide range of the sliding surface slopes.

3. There is given description of a patented bench model for determination of the hydrophoby degree of the materials and their hydraulic characteristics, as well as the results of an automated calculation of the Subcote FLP coating. The results of the experiments and the automated calculation data enabled comparison of the coefficients of hydraulic friction $\lambda$, which had been obtained on large- and small-sized benches and showed a high convergence of the results.

4. The possibility of application of small-sized benches instead of large-sized ones was proved for determination of hydrophoby and hydraulic parameters. In the future, it will greatly simplify the traditional method for comprehensive assessment of hydraulic parameters of various types of protective coatings and reduce time and financial costs of the experiments to be performed.

\section{References}

1. S. V. Khramenkov, O. G. Primin, Rekonstruktsiya truboprovodnyh system (2008)

2. A. Kuliczkowski, Rury kanalizacyjne (2004) 
3. Janssen A., 30 NO-DIG International Conference and Exhibition, 012287, 1-10 (2012)

4. Combeer G., Melville S. 31 NO-DIG International Conference and Exhibition, 1.19, 1-6 (2013)

5. D-H. Koo, C-H. Bae, J-H. Kim, T-J. Kweon 31 NO-DIG International Conference and Exhibition, 2013, 2.8, 1-6 (2013)

6. A. Kuliczkowski, E. Kuliczkowska, A. Zwierzchowska Technologie beswykopowe w inzeynierii srodowiska (2010)

7. Rabmer-Koller U. 29 NO-DIG International Conference and Exhibition, NO-DIG Berlin 2011, Paper 2C-1, pp. 1-10

8. Roscher H., J. Wasserwirt.-Wassertechn., 1-2, 36-40, 2007

9. G. N. Greaves, A. L. Greer, R. S. Lakes \& T. Rouxel, J. Nature Materials, 10, 823837 (2010)

10. Material safety data sheet 3M Scotchkote Rapid Setting Polymeric Lining 169HB // 3M Company, UK, 2012.

11. M. Rameil, Handbook of pipe bursting practice (2007)

12. S. Grossmann, D. Lohse, C, Eur. Phys. J. E., 40, 16 (2017)

13. M. I. Gorilovsky, J. Communal complex of Russia, 8 (122), 34-35 (2012)

14. M. I. Gorilovsky, Buyanovskii, V. M., S. J. Pliskin, A. N. Menshov, A. B., Branzburg, J. Plasticheskie MASSY, 8, 1-8 (2011)

15. V.A. Orlov, I.S. Dezhina, E.V. Orlov, I.A. Averkeev, Ispytatelniy stend po opredeleniyu stepeni hydrofobnosti materialov diya izgotovleniyq trub i remonta truboprovodov, Patent no.157695 (Russian Federation) (2015)

16. Sakai, M.; Song, J. H.; Yoshida, N.; Suzuki, S.; Kameshima, Y.; Nakajima, A. Langmuir 22, 4906-4909 (2006)

17. V.A. Orlov, S.P. Zotkin, I.S. Dezhina, A.A. Pelipenko, Programma rascheta stepeni hydrofobnosti i hydravlitcheskih parametrov trub i zaschitnyh pokrytiy, (2017) 